\title{
$\left(0.96 \mathrm{NaNbO}_{3}-0.04 \mathrm{CaZrO}_{3}\right)-x \mathrm{Fe}_{2} \mathrm{O}_{3}$ 反铁电 陶瓷的介电及储能性能研究
}

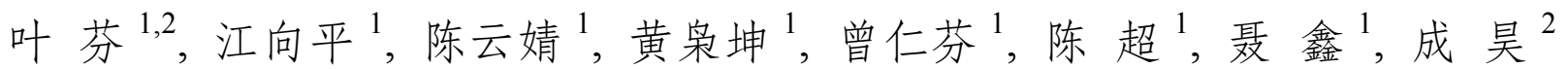
(1. 景德镇陶瓷大学 材料科学与工程学院, 江西省先进陶瓷材料重点实验室, 景德镇 333001; 2. 铜仁学院材料 与化学工程学院, 铜仁 554300)

摘 要: $0.96 \mathrm{NaNbO}_{3}-0.04 \mathrm{CaZrO}_{3}$ (简称 $\mathrm{NNCZ}$ ) 陶瓷在室温下展现出稳定的双电滞回线, 但是其储能密度、储能效率 和击穿强度都比较低, 限制其成为储能材料。本工作通过掺杂 $\mathrm{Fe}_{2} \mathrm{O}_{3}$, 利用 $\mathrm{Fe}^{3+}$ 离子变价的特点, 实现 NNCZ 储能 性能的优化。采用传统固相法制备了 $\left(0.96 \mathrm{NaNbO}_{3}-0.04 \mathrm{CaZrO}_{3}\right)-x \mathrm{Fe}_{2} \mathrm{O}_{3}$ (简称 $\mathrm{NNCZ}-x \mathrm{Fe}$ ) 反铁电储能陶瓷, 并对样品 的相结构、微观形貌、电学性能和储能性能进行了表征, 重点研究了 $\mathrm{Fe}_{2} \mathrm{O}_{3}$ 掺杂量对 NNCZ 陶瓷介电和储能性能的 影响规律。结果表明, 样品均具有单一的钙钋矿结构, 掺杂 $\mathrm{Fe}_{2} \mathrm{O}_{3}$ 能明显降低 NNCZ 陶瓷的烧结温度, 晶粒平均尺 寸随着掺杂量增大先减小后增大, 掺杂量 $x=0.02$ 时, 晶粒平均尺寸最小 $(5.04 \mu \mathrm{m})$, 且具有较好的储能性能。室温下, $\mathrm{NNCZ}-0.02 \mathrm{Fe}$ 击穿强度为 $230 \mathrm{kV} / \mathrm{cm}$, 击穿前的有效储能密度和储能效率分别为 $1.57 \mathrm{~J} / \mathrm{cm}^{3}$ 和 $55.74 \%$ 。在 $125{ }^{\circ} \mathrm{C}$ 和 外加电场 $180 \mathrm{kV} / \mathrm{cm}$ 下, NNCZ-0.02Fe 的储能密度为 $4.53 \mathrm{~J} / \mathrm{cm}^{3}$ 。掺杂 $\mathrm{Fe}_{2} \mathrm{O}_{3}$ 使 NNCZ 陶瓷的烧成温度降低, 氧空 位的迁移速率下降，抑制晶粒的长大，同时降低了介电损耗，使得击穿强度增加; 适量氧空位钉扎使得反铁电相向 铁电相相翻转变得困难, 避免出现哑铃状双电滞回线, 从而提高储能效率。本研究结果表明 NNCZ- $x \mathrm{Fe}$ 在电介质储 能领域具有潜在应用价值。

关 键 词: $\mathrm{NaNbO}_{3}$; 反铁电材料; 储能性能; 介电性能

中图分类号: TQ174 文献标志码: A

\section{Dielectric and Energy Storage Property of $\left(0.96 \mathrm{NaNbO}_{3}-0.04 \mathrm{CaZrO}_{3}\right)-x \mathrm{Fe}_{2} \mathrm{O}_{3}$ Antiferroelectric Ceramics}

\author{
YE Fen $^{1,2}$, JIANG Xiangping ${ }^{1}$, CHEN Yunjing ${ }^{1}$, HUANG Xiaokun ${ }^{1}$, \\ ZENG Renfen ${ }^{1}$, CHEN Chao ${ }^{1}$, NIE Xin ${ }^{1}$, CHENG Hao ${ }^{2}$
}

(1. Jiangxi Key Laboratory of Advanced Ceramic Materials, School of Material Science and Engineering, Jingdezhen Ceramic Institute, Jingdezhen 333001, China; 2. College of Material and Chemical Engineering, Tongren University, Tongren 554300, China)

Abstract: $0.96 \mathrm{NaNbO}_{3}-0.04 \mathrm{CaZrO}_{3}(\mathrm{NNCZ})$ ceramic shows stable double hysteresis loops at room temperature, but the property of energy density, energy storage efficiency and breakdown strength of NNCZ are terrible, which limit

收稿日期：2021-06-28; 收到修改稿日期：2021-07-20; 网络出版日期：2021-10-21

基金项目: 国家自然科学基金(52062018, 51862016, 51762024); 江西省自然科学基金(20192BAB20600, 20192BAB212002); 江西省教育厅科技项目(GJJ190712, GJJ190699); 贵州省教育厅创新团队(KY[2018]030)

National Natural Science Foundation of China (52062018, 51862016, 51762024); Natural Science Foundation of Jiangxi Province (20192BAB20600, 20192BAB212002); Foundation of Jiangxi Provincial Education Department (GJJ190712, GJJ190699); Foundation of the Department of Education of Guizhou Province (KY[2018]030)

作者简介: 叶 芬(1987-), 女, 博士研究生. E-mail: yefen1987@163.com YE Fen (1987-), female, PhD candidate. E-mail: yefen1987@163.com

通信作者: 江向平, 教授.E-mail: jiangxp64@163.com; 成 昊, 教授. E-mail: smallone.1@163.com JIANG Xiangping, professor. E-mail: jiangxp64@163.com; CHENG Hao, professor. E-mail: smallone.1@163.com 
$\mathrm{NNCZ}$ to be used as energy storage materials. In this work, $\mathrm{Fe}_{2} \mathrm{O}_{3}$ was chosen to modify the energy storage property of NNCZ. $\left(0.96 \mathrm{NaNbO}_{3}-0.04 \mathrm{CaZrO}_{3}\right)-x \mathrm{Fe}_{2} \mathrm{O}_{3}(\mathrm{NNCZ}-x \mathrm{Fe})$ antiferroelectric ceramics were prepared by traditional solid reaction method. The phase, morphology, dielectric property and energy storage property of NNCZ-xFe were characterized. The results indicated that the crystal structures of NNCZ-xFe ceramics were pure perovskite structure. The sintering temperature of NNCZ ceramic was decreased with addition of $\mathrm{Fe}_{2} \mathrm{O}_{3}$. With the increase of $\mathrm{Fe}_{2} \mathrm{O}_{3}$ content, the grain size of NNCZ-xFe was decreased firstly and then raised. The NNCZ-0.02Fe ceramic obtained the smallest grain size $(5.04 \mu \mathrm{m})$ and the best energy storage property. The breakdown strength of NNCZ-0.02Fe was $230 \mathrm{kV} / \mathrm{cm}$ at room temperature (RT). The recoverable energy density and energy storage efficiency before breakdown were $1.57 \mathrm{~J} / \mathrm{cm}^{3}$ and $55.74 \%$ respectively. At $125{ }^{\circ} \mathrm{C}$ and $180 \mathrm{kV} / \mathrm{cm}$, the energy density of NNCZ$0.02 \mathrm{Fe}$ was $4.53 \mathrm{~J} / \mathrm{cm}^{3} . \mathrm{Fe}_{2} \mathrm{O}_{3}$ doping decreased the sintering temperature of NNCZ ceramics, reduced the the migration rate of oxygen vacancies and inhibited the growth of grains. At the same time, it reduced the dielectric loss and improved the breakdown strength. The oxygen vacancies pinning made antiferroelectric phase switch to ferroelectric phase harder, avoided appearance of dumbbell-shaped double hysteresis loops, so the energy storage efficiency was improved. This research shows that NNCZ- $x$ Fe has a good potential application in the field of dielectric energy storage.

Key words: $\mathrm{NaNbO}_{3}$; antiferroelectric material; energy storage property; dielectric property

介质电容器因具有储能密度大、温度稳定性 好、充放电效率高等优点而被应用于先进电器设备 领域 ${ }^{[1-3]}$ 。作为介质电容器核心材料的反铁电 (Antiferroelectric, AFE) 材料因具备双电滞回线特性, 当外加电场升至铁电相畴翻转 $\left(E_{\mathrm{AF}}\right)$ 时, 介电常数增 加 ${ }^{[4]}$, 比线性电介质和铁电电介质储存和释放更多 的电能 ${ }^{[5]} 。 1949$ 年, $\mathrm{NaNbO}_{3}(\mathrm{NN})$ 晶体被 Matthias ${ }^{[6]}$ 发现, 并认为是铁电 (Ferroelectric, FE) 材料, 之后 Vousden ${ }^{[7]}$ 发现 $\mathrm{NaNbO}_{3}(\mathrm{NN})$ 在室温下表现出 $\mathrm{AFE}$ 特性。

目前, 有关 NN 陶瓷晶相组成调控的研究报道 很多 ${ }^{[8-9]}$, 如利用压力、温度、外置电场、晶粒尺寸 和化学组成等方法调控, 普遍认为, $N N$ 陶瓷在室温 下的主晶相为 $\operatorname{AFE}\left(\mathrm{P}\right.$ 相), 亚稳相为 $\operatorname{FE}(\mathrm{Q} \text { 相 })^{[4]}$, 尺 寸效应和外加电场可以诱导 $\mathrm{NN}$ 陶瓷出现亚稳 $\mathrm{FE}$ 相 ${ }^{[8]}$, 所以 $\mathrm{NN}$ 陶瓷很难在室温下观察到双电滞回 线, 从而限制了 $\mathrm{NN}$ 陶瓷在储能领域的应用。美国 宾夕法尼亚州立大学 CLIVE 课题组 ${ }^{[4,9-13]}$ 通过掺杂 $\left(\mathrm{BiScO}_{3}(\mathrm{BS}) 、 \mathrm{SrZrO}_{3}(\mathrm{SZ}) 、 \mathrm{CaZrO}_{3}(\mathrm{CZ}) 、 \mathrm{CaHfO}_{3}(\mathrm{CH})\right)$ 使 NN 出现了双电滞回线, 但是没有对储能性能进 行评价。 $\mathrm{Liu}$ 等 ${ }^{[2]}$ 研究了 $\mathrm{CZ}$ 掺杂对 $\mathrm{NN}$ 微观结构、 相组成和电学性能的影响, 成功获得了双电滞回线, 当 $\mathrm{CZ}$ 掺杂量(摩尔分数)为 $0.04 \%$ 时, $\mathrm{NN}-0.04 \mathrm{CZ}$ 陶 瓷晶粒平均粒径最小, 击穿强度 (Breakdown strength, BDS) 约 $141 \mathrm{kV} / \mathrm{cm}$, 有效储能密度 $\left(W_{\mathrm{rec}}\right)$ 只有 $0.55 \mathrm{~J} / \mathrm{cm}^{3}$, 储能效率 $(\eta)$ 仅为 $63 \%$, 与其它 AFE材料 的储能性能相差较大, 且 BDS 较低。Qi 等 ${ }^{[14]}$ 研究
了 $(0.94-x) \mathrm{NaNbO}_{3}-0.06 \mathrm{BaZrO}_{3}-x \mathrm{CaZrO}_{3}$ 陶瓷的储能 性能, 当 $x=0.04$ 时, 在 $0.1 \mathrm{~Hz}$ 下, 样品的 $W_{\mathrm{rec}}$ 为 $1.59 \mathrm{~J} / \mathrm{cm}^{3}$, 但 $\eta$ 只有 $30 \%$ 。Wang 课题组 ${ }^{[15-16]}$ 通过 掺杂 $\mathrm{CaSnO}_{3}(\mathrm{CS})$, 在室温下成功获得了双电滞回线, 并研究了稀土 $\mathrm{La} 、 \mathrm{Sm}$ 和 $\mathrm{Lu}$ 对 $0.96 \mathrm{NN}-0.04 \mathrm{CS}$ 储能 性能的影响。

储能密度 $(W)$ 、有效储能密度 $\left(W_{\mathrm{rec}}\right)$ 和储能效率 $(\eta)$ 是储能材料的主要参数, 计算公式如下 ${ }^{[1]}$ :

$$
\begin{gathered}
W=\int_{0}^{P_{\max }} E \mathrm{~d} P \\
W_{\mathrm{rec}}=\int_{P_{\mathrm{r}}}^{P_{\max }} E \mathrm{~d} P \\
\eta=\frac{W_{\mathrm{rec}}}{W} \times 100 \%
\end{gathered}
$$

其中, $P, P_{\max }, P_{\mathrm{r}}$ 和 $E$ 分别代表极化强度、最大极化 强度、剩余极化强度和外加电场强度。为了提高 $W$ 和 $W_{\mathrm{rec}}$, 需要同时增加 $P_{\max }$, 减小 $P_{\mathrm{r}}$ 并提高 $\mathrm{BDS}$ 。

变价离子价态丰富, 可以占据 $\mathrm{A}$ 位、 $\mathrm{B}$ 位或者 同时占据 $\mathrm{A}$ 位和 $\mathrm{B}$ 位, 能够很好地调控材料的性 能。如 $\mathrm{Mn}$ 离子在煅烧过程中可能出现 $\mathrm{Mn}^{2+} / \mathrm{Mn}^{3+} / \mathrm{Mn}^{4+}$, 对应的离子半径为 $0.083 、 0.064$ 和 $0.053 \mathrm{~nm}$, 可以取代 $\mathrm{A}$ 位的 $\mathrm{Ag}$ 或者 $\mathrm{B}$ 位的 $\mathrm{Nb}$, 对 $\mathrm{AgNbO}_{3}$ 的储能性能起到很好的调控作用 ${ }^{[17]}$ 。掺 杂 $\mathrm{Mn}$ 减少了 $\mathrm{NN}$ 陶瓷的氧空位, 电学性能得到改 善 ${ }^{[18]}$ 。本工作采用固相法制备了 $\mathrm{NNCZ}-x \mathrm{Fe}$ 陶瓷, 通过掺杂可变价的过渡金属元素 $\mathrm{Fe}$ 来调控 $0.96 \mathrm{NaNbO}_{3}-0.04 \mathrm{CaZrO}_{3}(\mathrm{NNCZ})$ 陶瓷性能, 以降低 
NNCZ 的烧结温度, 减小样品的晶粒尺寸, 抑制氧 空位的迁移速率, 提高其 BDS, 从而最终提高 $\mathrm{NNCZ}$ 陶瓷的 $W_{\mathrm{rec}}$ 和 $\eta$ 。

\section{1 实验方法}

采用固相法制备 $\left(0.96 \mathrm{NaNbO}_{3}-0.04 \mathrm{CaZrO}_{3}\right)$ $x \mathrm{Fe}_{2} \mathrm{O}_{3}(x=0,0.005,0.01,0.015,0.02,0.025)$ 陶瓷, 简 写 NNCZ- $x \mathrm{Fe}$ 。 $\mathrm{Nb}_{2} \mathrm{O}_{5}(99.5 \%) 、 \mathrm{Na}_{2} \mathrm{CO}_{3}(99.8 \%)$ 、 $\mathrm{CaCO}_{3}(99 \%) 、 \mathrm{ZrO}_{2}(99 \%) 、 \mathrm{Fe}_{2} \mathrm{O}_{3}(99 \%)$ 称量前充分 干燥, 以无水乙醇为分散剂, 氧化锆球为介质, 球 磨 $24 \mathrm{~h}$, 料浆烘干后压成直径为 $5 \mathrm{~cm}$ 的圆片, 在 $800{ }^{\circ} \mathrm{C}$ 下预烧 $6 \mathrm{~h}$ 。二次球磨后的泥浆干燥过篮得到 预合成的粉体, 加入 PVA(质量分数 $5 \%$ ) 造粒, 在 $340 \mathrm{MPa}$ 下压制成直径为 $13 \mathrm{~mm}$, 厚度 $1 \mathrm{~mm}$ 圆片 生坏。在 $600{ }^{\circ} \mathrm{C}$ 排胶 $2 \mathrm{~h}$, 采用埋烧的方式, NNCZ 和 NNCZ- $x \mathrm{Fe}$ 分别在 1370 和 $1230{ }^{\circ} \mathrm{C}$ 下煅烧 $2 \mathrm{~h}$ 得 到陶瓷。陶瓷样品两面经过抛光, 使用丝网印刷, 根 据测试需要刷不同直径的银浆, 在 $550{ }^{\circ} \mathrm{C}$ 下保温 $30 \mathrm{~min}$ 制备银电极, 用于电学性能测试。

采用 $\mathrm{X}$ 射线衍射仪(XRD, D8-Advance A25, Bruker)和扫描电子显微镜(SEM, SU8010, Hitachi) 对样品相组成和微观形貌进行表征, 采用介电温谱 测试系统(HP4294A, Agilent)测试介电温谱, 采用铁 电综合测试系统(Trek model 609B, Radiant) 获得陶 瓷的电滞回线, 采用 $\mathrm{X}$ 射线光电子能谱仪 (XPS, EscaLab250Xi, Thermo Fisher Scientific)对样品表面 的化学元素价态及含量进行表征, 采用绝缘电阻仪 测得直流电阻(TH2684, Tonghui)。

\section{2 结果与讨论}

图 1 为 NNCZ- $x \mathrm{Fe}$ 陶瓷的 XRD 图谱。由图 1(a) 可见, NNCZ- $x \mathrm{Fe}$ 陶瓷呈现单一的钙钛矿相, 没有出 现明显的第二相, 且结晶度较好, 说明 $\mathrm{Fe}^{3+}$ 成功固 溶进入 $\mathrm{NNCZ}$ 晶格。从局部放大的图 1(b,c) 可见，随 着 $\mathrm{Fe}_{2} \mathrm{O}_{3}$ 掺杂量增大, 衍射峰先向低角度再向高角 度移动, 表明 NNCZ- $x \mathrm{Fe}$ 陶瓷晶胞体积随着 $\mathrm{Fe}_{2} \mathrm{O}_{3}$ 掺杂量的增加, 先变大后减小。 $\mathrm{NNCZ}$ 的烧结温度 为 $1370{ }^{\circ} \mathrm{C}$, 在较高温度下 $\mathrm{Na}^{+}$会挥发, 从而导致化学 计量偏离和晶粒异常长大及晶胞体积减小 ${ }^{[19]}$ 。掺杂 $\mathrm{Fe}_{2} \mathrm{O}_{3}$ 使 $\mathrm{NNCZ}$ 陶瓷的烧结温度降到 $1230{ }^{\circ} \mathrm{C}$, 烧结 温度降低使钠离子挥发量减少, 与 $\mathrm{NNCZ}$ 相比, 晶胞 体积变大。所以少量离子半径较小的 $\mathrm{Fe}^{3+}(0.0645 \mathrm{~nm})$ 置换离子半径较大的 $\mathrm{Zr}^{4+}(0.072 \mathrm{~nm}), \mathrm{NNCZ}-0.005 \mathrm{Fe}$
陶瓷的晶胞体积仍然比 NNCZ 大, XRD 衍射峰向低 角度偏移 ${ }^{[20]}$ 。

众所周知, 离子半径差小于 $15 \%$, 离子价态相 等时容易形成置换固溶体。 $\mathrm{Fe}^{3+}$ 与 $\mathrm{Zr}^{4+}(0.072 \mathrm{~nm})$ 离 子半径差为 $11.63 \%, \mathrm{Fe}^{3+}$ 与 $\mathrm{Zr}^{4+}$ 价态最接近, 掺杂的 $\mathrm{Fe}^{3+}$ 优先与 $\mathrm{Zr}^{4+}$ 发生置换, 再与 $\mathrm{Nb}^{5+}(0.064 \mathrm{~nm})$ 发生 置换。随着 $\mathrm{Fe}_{2} \mathrm{O}_{3}$ 掺杂量进一步增大, 离子半径小 的 $\mathrm{Fe}^{3+}$ 置换了更多离子半径大的 $\mathrm{Zr}^{4+}$, 使得 $\mathrm{NNCZ}$ $x \mathrm{Fe}$ 陶瓷的 $<\mathrm{B}-\mathrm{O}>$ 八面体体积进一步减小, 导致晶 胞体积减小 ${ }^{[21]}, \mathrm{XRD}$ 衍射峰逐渐向高角度偏移, 如 图 1(b, c) 所示。此外, 质量较小的 $\mathrm{Fe}^{3+}$ 离子取代较 大的 $\mathrm{Zr}^{4+}$ 离子, 会使拉曼模向高频方向移动, 进一 步证明 $\mathrm{Fe}^{3+}$ 已进入晶格。

图 2 是 NNCZ- $x \mathrm{Fe}$ 陶瓷的表面形貌照片。从 图 2(a)可知, NNCZ 陶瓷晶粒尺寸大小不均匀且有 异常长大的现象, 与 $\mathrm{Liu}$ 等 $^{[2]}$ 研究结果一致。掺杂 $\mathrm{Fe}_{2} \mathrm{O}_{3}$ 后，样品的煅烧温度降低，晶粒尺寸明显减小， 如图 2(b)所示。采用 Nano Measurer 软件统计了 $\mathrm{NNCZ}-x \mathrm{Fe}$ 陶瓷晶粒的平均尺寸，如图 2(c)所示，随 着 $\mathrm{Fe}_{2} \mathrm{O}_{3}$ 掺杂量增大, $\mathrm{NNCZ}-x \mathrm{Fe}$ 陶瓷晶粒的平均尺 寸先减小后增大, 当 $x=0.02$ 时, 平均晶粒尺寸最小 为 $5.04 \mu \mathrm{m}$ 。

$\mathrm{NNCZ}$ 陶瓷的氧空位主要来源于高温㷽烧时 $\mathrm{Na}^{+}$的挥发, 为了平衡价态, 产生了氧空位, 大量的 氧空位为离子迁移提供了通道, 促进了晶粒的生 $k^{[2]}$ 。 NNCZ- $x \mathrm{Fe}$ 的氧空位一部分来源于 $\mathrm{Na}^{+}$挥发, 另外一部分来源于低价态的 $\mathrm{Fe}^{3+}$ 置换了高价态的 $\mathrm{Zr}^{4+}$ 和 $\mathrm{Nb}^{5+}$ 。掺杂 $\mathrm{Fe}_{2} \mathrm{O}_{3}$ 使煅烧温度明显降低, $\mathrm{Na}^{+}$ 的挥发量减少, 与 $\mathrm{NNCZ}$ 相比, 该部分氧空位数量下 降。 $\mathrm{Fe}_{2} \mathrm{O}_{3}$ 在高温情况下容易还原成 $\mathrm{FeO}$, 产生游离 的氧 $[\mathrm{O}]$, 见式(4) ${ }^{[20]}$, 产生的 $[\mathrm{O}]$ 会消耗一部分氧空
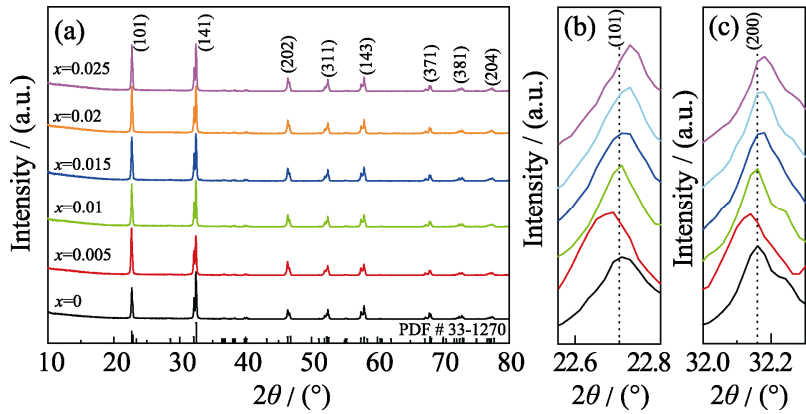

图 $1 \mathrm{NNCZ}-x \mathrm{Fe}$ 陶瓷的 XRD 图谱(a), 22.5 $\sim 22.8^{\circ}$ 放大图谱 (b)和 $31.8^{\circ} \sim 33.0^{\circ}$ 放大图谱 $(c)$

Fig. 1 XRD patterns of NNCZ- $x$ Fe ceramics (a), enlarged XRD patterns from $22.5^{\circ}$ to $22.8^{\circ}$ (b), and from $31.8^{\circ}$ to $33.0^{\circ}$ (c) of NNCZ- $x \mathrm{Fe}$ ceramics 


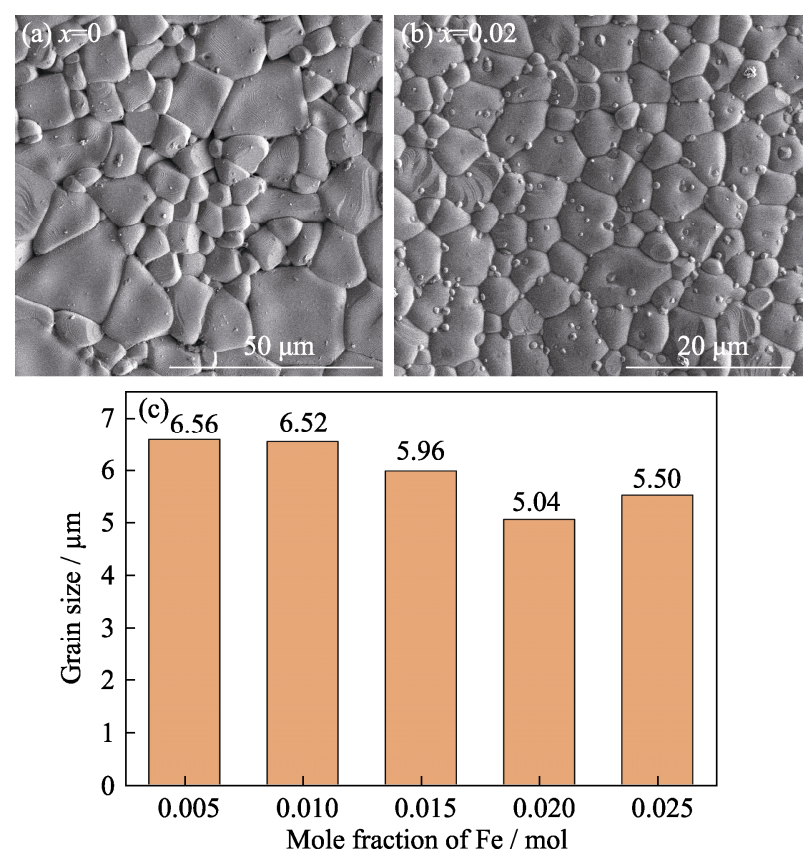

图 $2 \mathrm{NNCZ}-x \mathrm{Fe}$ 陶瓷的表面形貌照片及其平均粒径

Fig. 2 Surface morphologies of NNCZ- $x \mathrm{Fe}$ ceramics and corresponding average grain size

(a) $x=0$; (b) $x=0.02$; (c) Average grain size

位, 所以适量掺杂 $\mathrm{Fe}_{2} \mathrm{O}_{3}$ 不会引起 $\mathrm{NNCZ}$ 陶瓷氧空 位数量发生较大变化。当掺入过量 $\mathrm{Fe}_{2} \mathrm{O}_{3}$ 时, 氧空位 数量进一步增加, 促进晶粒生长。所以当掺杂量增加 到 $0.025 \%$ 时，晶粒平均尺寸有上升趋势，见图 2(c)。

$$
\mathrm{Fe}_{2} \mathrm{O}_{3} \rightarrow 2 \mathrm{FeO}+[\mathrm{O}]
$$

进一步研究 $\mathrm{Fe}_{2} \mathrm{O}_{3}$ 掺杂对 NNCZ 陶瓷氧空位数 量的影响, 对 NNCZ 和 NNCZ-0.02Fe 陶瓷样品的 $\mathrm{O} 1 \mathrm{~s}$ 进行了 XPS 分析, 如图 3 所示。 $\mathrm{NNCZ}$ 可以拟 合出 3 个 Gauss 峰, $\mathrm{O}_{\mathrm{L}}$ 在 $529 \mathrm{eV}$ 附近, 对应的是钙 钛矿结构的晶格氧。图中其它峰是与 $\mathrm{OH}$ 基相关的 吸附氧, 一般通过检测陶瓷表面 $\mathrm{OH}$ 含量, 可以间 接表征氧空位浓度 ${ }^{[2]}$ 。 $\mathrm{O}_{\mathrm{V}}$ 在 $531 \mathrm{eV}$ 附近, 对应氧空 位; $\mathrm{O}_{\mathrm{w}}$ 在 $533 \mathrm{eV}$ 附近, 对应吸附水 ${ }^{[22-23]}$ 。掺杂 $\mathrm{Fe}_{2} \mathrm{O}_{3}$ 使 NNCZ 陶瓷表面 $\mathrm{O}$ 的形态多样化, 拟合出了第四 个峰 $\left(\mathrm{O}_{\mathrm{Fe}}\right)$, 这个峰与 $\mathrm{Fe}^{3+}$ 有关 ${ }^{[24]}$ 。一般通过氧空位 与晶格氧之间的面积比 $\left(\mathrm{O}_{\mathrm{V}} / \mathrm{O}_{\mathrm{L}}\right)$ 可以间接判断氧空 位浓度的变化, 由图 3 可知, NNCZ 和 NNCZ-0.02Fe 的 $\mathrm{O}_{\mathrm{V}} / \mathrm{O}_{\mathrm{L}}$ 分别为 0.41 和 0.45 , 因此 $\mathrm{NNCZ}$ 和 $\mathrm{NNCZ}-0.02 \mathrm{Fe}$ 氧空位浓度比较接近。

就压电陶瓷而言, 中低温条件下最可能发生迁 移的是氧空位, 氧空位浓度和迁移速率是影响其电 学性能的重要因素。NNCZ 和 NNCZ-0.02Fe 陶瓷氧 空位浓度接近, 故氧空位的迁移速率是影响其电学 性能的主要因素, 可以间接通过氧空位激活能 $\left(E_{\mathrm{a}}\right)$
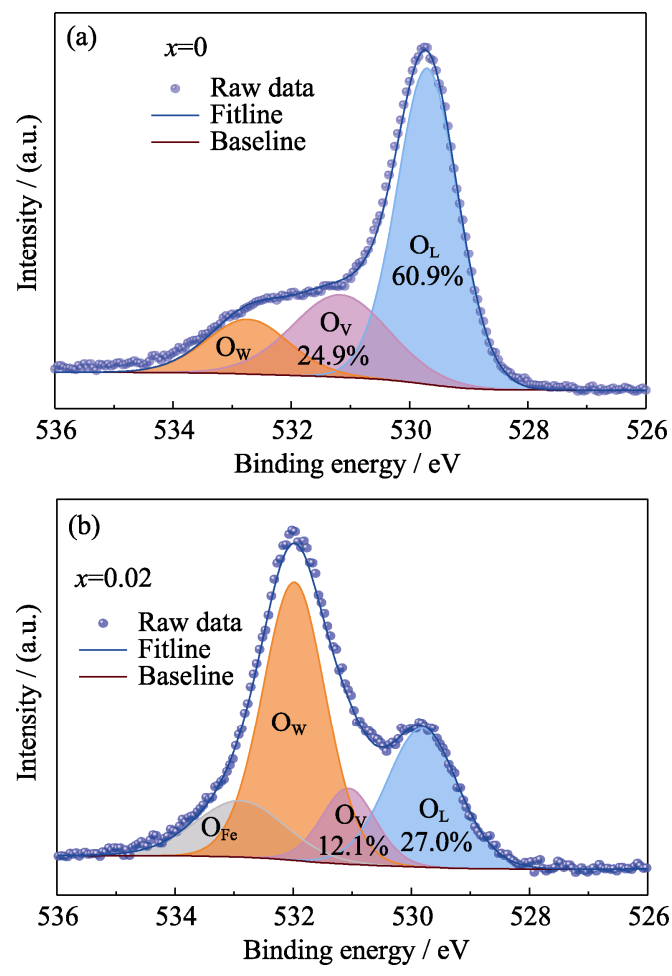

图 $3 \mathrm{NNCZ}-x \mathrm{Fe}$ 陶瓷的 O1s XPS 图谱

Fig. 3 XPS spectra of O1s peak for NNCZ- $x \mathrm{Fe}$ (a) $x=0$; (b) $x=0.02$

和电阻率 $(\rho)$ 来表征。对 $\mathrm{NNCZ}$ 和 $\mathrm{NNCZ}-0.02 \mathrm{Fe}$ 陶 瓷晶粒和晶界的 $E_{\mathrm{a}}$ 分别进行拟合计算 ${ }^{[25-26]}, E_{\mathrm{a}}$ 在 $0.74 \sim 1.14 \mathrm{eV}$ 范围, 表明在 $350 \sim 470{ }^{\circ} \mathrm{C}$ 范围内 $\mathrm{NNCZ}$ 和 NNCZ-0.02Fe 陶瓷均以氧空位电导为主 ${ }^{[27]}$ 。 $\mathrm{NNCZ}$ 和 $\mathrm{NNCZ}-0.02 \mathrm{Fe}$ 晶粒的 $E_{\mathrm{a}}$ 接近但是低于晶 界, 说明晶界是影响氧空位迁移速率的主要因素。 $\mathrm{NNCZ}-0.02 \mathrm{Fe}$ 陶瓷的晶界 $E_{\mathrm{a}}$ 高于 $\mathrm{NNCZ}$ 陶瓷, 表明 氧空位在 $N N C Z-0.02 \mathrm{Fe}$ 陶瓷内的迁移速率低于 $\mathrm{NNCZ}$ 陶瓷。根据以上分析可知 NNCZ-0.02Fe 陶瓷 的 $\rho$ 应高于 NNCZ 陶瓷, NNCZ-0.02Fe 的实测 $\rho$ 确

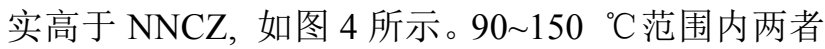
电阻率相差较大, 随着温度升高, 两者差距逐渐缩 小。氧空位容易与缺陷形成缺陷偶极子对 $\left(\mathrm{Fe}_{\mathrm{Zr}}^{\prime}-\mathrm{V}_{\mathrm{O}}^{\bullet \bullet}\right.$ 或 $\left.\mathrm{Fe}_{\mathrm{Nb}}^{\prime \prime}-\mathrm{V}_{\mathrm{O}}^{\bullet \bullet}\right)$ 限制氧空位的迁移 ${ }^{[21]}$, 使 $\rho$ 升高, 另外, $\mathrm{NNCZ}-0.02 \mathrm{Fe}$ 陶瓷晶粒尺寸较小, 晶界密度大, 使 氧空位迁移变得更困难。

图 5(a,b)为 NNCZ- $x \mathrm{Fe}$ 陶瓷在 $100 \mathrm{kHz}$ 频率下 测得的介电常数 $\varepsilon_{\mathrm{r}}$ 和介电损耗 $\tan \delta$ 随温度变化的曲 线图, 图 5(c) 为 NNCZ- $x \mathrm{Fe}$ 的居里温度 $\left(T_{\mathrm{C}}\right)$ 及室温下 的介电常数与 $\mathrm{Fe}_{2} \mathrm{O}_{3}$ 掺杂量的关系曲线。在 $300{ }^{\circ} \mathrm{C}$ 左右出现的介电异常峰, 对应于 AFE 的 P 相向 R 相 $(\mathrm{Pnmm})$ 转变 ${ }^{[15]}$, 如图 5(a)所示。随着 $\mathrm{Fe}_{2} \mathrm{O}_{3}$ 掺杂量 

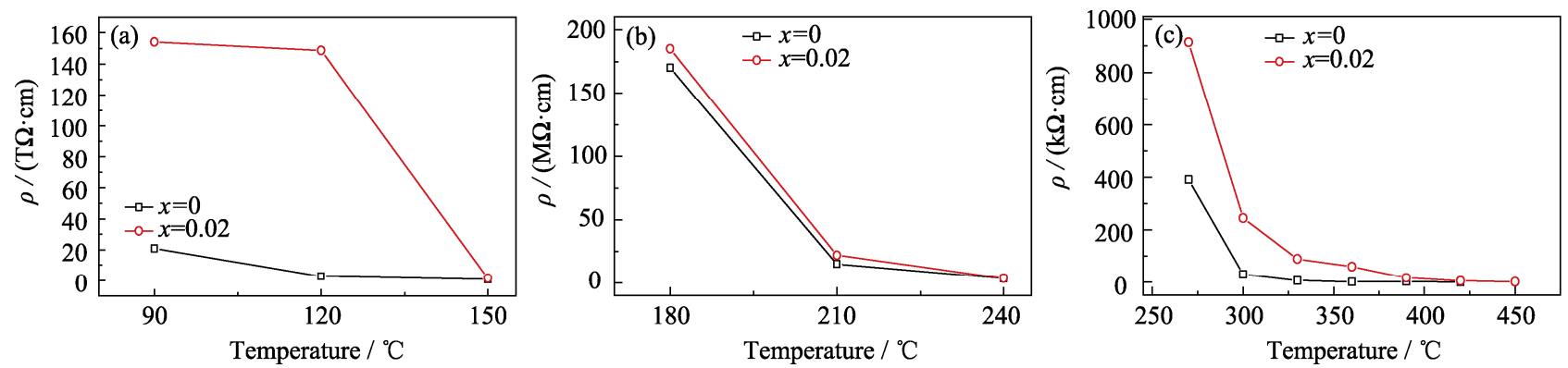

图 $4 \mathrm{NNCZ}-x \mathrm{Fe}$ 陶瓷的电阻率 $(\rho)$ 随温度的变化曲线

Fig. 4 Temperature dependence of electrical resistivity $(\rho)$ for NNCZ- $x \mathrm{Fe}$ in the different temperature range

变化, NNCZ 的介电损耗也发生变化, 由图 5(b)可知, $x=0.02$ 样品的介电损耗最低, 这是由于 NNCZ-0.02Fe 氧空位的迁移速率较低, 晶界较多, 损耗相应减小。 从图 5(c)可知, $\mathrm{NNCZ}-x \mathrm{Fe}$ 陶瓷的 $T_{\mathrm{C}}$ 比较接近。随着 $\mathrm{Fe}_{2} \mathrm{O}_{3}$ 掺杂量增大, 介电常数先增大后减小。这是由 于较大离子极化率的 $\mathrm{Fe}^{3+}\left(2.14 \times 10^{-3} \mathrm{~nm}^{3}\right)$ 置换了离 子极化率较小的 $\mathrm{Zr}^{4+}\left(2.023 \times 10^{-3} \mathrm{~nm}^{3}\right)$, 所以介电常 数上升; 当过量掺杂时, $\mathrm{Fe}^{3+}$ 置换了离子极化率较高 的 $\mathrm{Nb}^{5+}\left(3.1 \times 10^{-3} \mathrm{~nm}^{3}\right)$, 介电常数又随之下降 ${ }^{[28]}$ 。

NNCZ- $x$ Fe 陶瓷在室温下测得的电滞回线如图 6 所示, 样品均表现出双电滞回线。 $x=0.005 \sim 0.02$ 样品 均表现出夹紧状双电滞回线, 当掺杂量增加到 $x=0.025$ 时, 出现哑铃状的双电滞回线, 且 $P_{\max }$ 明显 增大。从 NNCZ- $x \mathrm{Fe}$ 陶瓷的电流回线可以看到, 掺 杂后的样品均出现了 4 个电流峰, 且 $\mathrm{AFE} \rightarrow \mathrm{FE}$ 对应 的电流峰明显, 表明 NNCZ- $x$ Fe 陶瓷均以 AFE 的微 米畴为主 ${ }^{[16]}$ 。且随着掺杂量增大, $\mathrm{AFE} \rightarrow \mathrm{FE}$ 对应的 电流峰逐渐明显, 表明样品中 $\mathrm{FE}$ 相增多, 所以 $P_{\max }$ 逐渐增大。 $x=0.005 、 0.01 、 0.015 、 0.02$ 和 0.025 样 品对应的 $\mathrm{AFE} \rightarrow \mathrm{FE}$ 翻转电场强度 $\left(E_{\mathrm{AF}}\right)$ 分别为: $202.2 、 200.1 、 198.9 、 198.4$ 和 $185.2 \mathrm{kV} / \mathrm{cm}$, 过量的 $\mathrm{Fe}_{2} \mathrm{O}_{3}$ 使 $E_{\mathrm{AF}}$ 减小。

氧空位对铁电畴能起到钉扎作用, 但是钉扎效
应与氧空位数量存在最优配比。掺杂过量 $\mathrm{Fe}_{2} \mathrm{O}_{3}$ 使 $\mathrm{NNCZ}$ 陶瓷氧空位进一步增加, 钉扎效应减弱, 使 得 NNCZ-0.025Fe 陶瓷中大量的 AFE 向 FE 转变, 出 现如图 6(f)所示的哑铃状双电滞回线, $P_{\text {max }}$ 明显增大。

$\mathrm{NNCZ}-x \mathrm{Fe}$ 陶瓷的击穿强度 (BDS)及击穿前的 $W_{\text {rec }}$ 和 $\eta$ 如图 7(a,b) 所示, 随着掺杂量增大, BDS 先 增加后减小。影响 BDS 的因素很多, 如带隙、气孔、 粒径、第二相和厚度等。介电陶瓷发生击穿的形式 有本征击穿、电子击穿、热击穿和缺陷击穿 ${ }^{[29]}{ }_{0} \mathrm{Fe}_{2} \mathrm{O}_{3}$ 掺杂后, NNCZ- $x \mathrm{Fe}$ 的晶粒尺寸减小, 小而多的晶界 可以作为移动电荷的耗尽区, 氧空位的迁移速率小 使介电损耗降低, 加电压时降低了热击穿概率, 所 以 $\mathrm{Fe}_{2} \mathrm{O}_{3}$ 掺杂使 $\mathrm{NNCZ}$ 陶瓷的 BDS 升高, 当 $x=0.02$ 时 $\mathrm{BDS}$ 出现了最大值, 对应的 $W_{\mathrm{rec}}$ 和 $\eta$ 分别为 $1.57 \mathrm{~J} / \mathrm{cm}^{3}$ 和 $55.74 \%$ 。

$\mathrm{NNCZ}-x \mathrm{Fe}$ 陶瓷的 $W_{\mathrm{rec}}$ 和 $\eta$ 随着电场的变化曲 线如图 7(c, d) 所示, 随着电场强度增大, $W_{\mathrm{rec}}$ 逐渐增 大, 掺杂 $\mathrm{Fe}_{2} \mathrm{O}_{3}$ 明显提高了 $\mathrm{NNCZ}$ 的 $\eta$ 。在 $200 \mathrm{kV} / \mathrm{cm}$ 电场强度下, $x=0.02$ 和 $x=0.025$ 样品的 $W$ 和 $W_{\mathrm{rec}}$ 分别 为 $2.35 、 1.36$ 和 $3.32 、 1.39 \mathrm{~J} / \mathrm{cm}^{3}$, 但 $x=0.025$ 样品 的 $\eta$ 明显恶化, 作为储能陶瓷不仅需要较高的 $W$, 也需要较稳定的 $\eta$, 避免材料在使用过程中过早损 坏 ${ }^{[30]}$ 。 $x=0.02$ 样品的 $\eta$ 相对其它样品较稳定, 所以
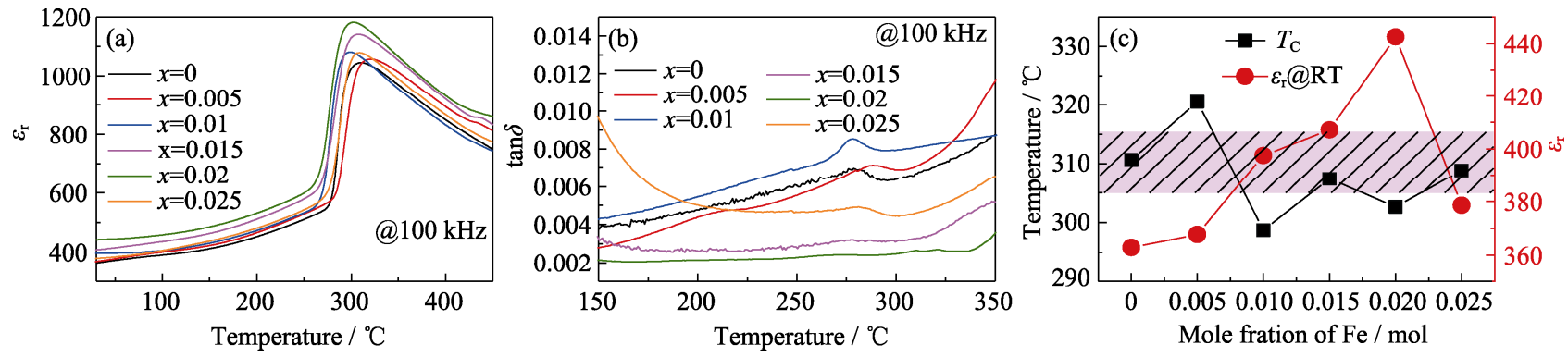

图 $5 \mathrm{NNCZ}-x \mathrm{Fe}$ 陶瓷的介电性能

Fig. 5 Dielectric property of NNCZ- $x \mathrm{Fe}$ ceramics

(a) Temperature dependence of relative permittivity of NNCZ- $x \mathrm{Fe}$ ceramics; (b) Loss tangent of NNCZ- $x \mathrm{Fe}$ ceramics; (c) Change of Curie temperature $\left(T_{\mathrm{C}}\right)$ and dielectric constant at room temperature (RT) with the content of $\mathrm{Fe}^{3+}$

colorful figures are available on website 

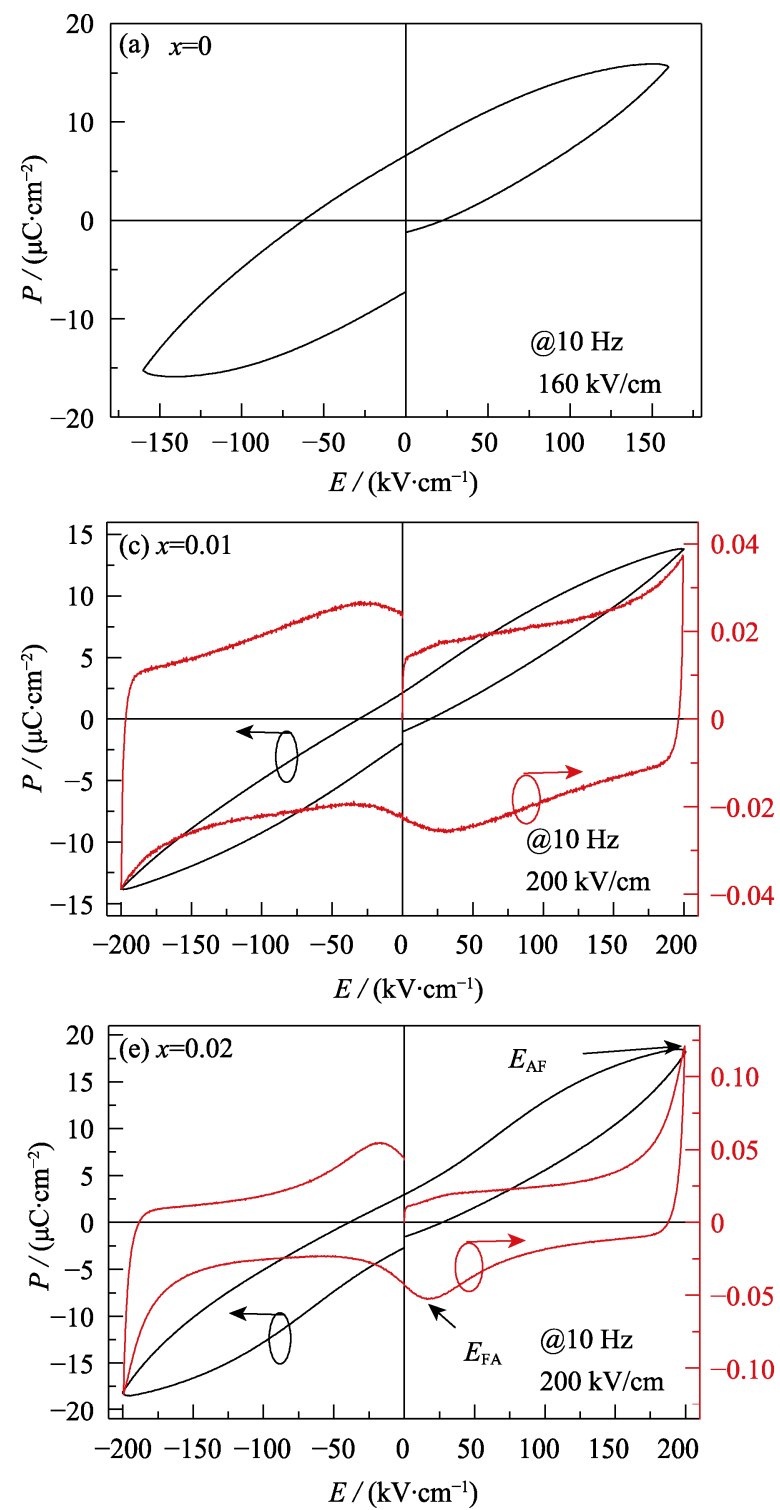
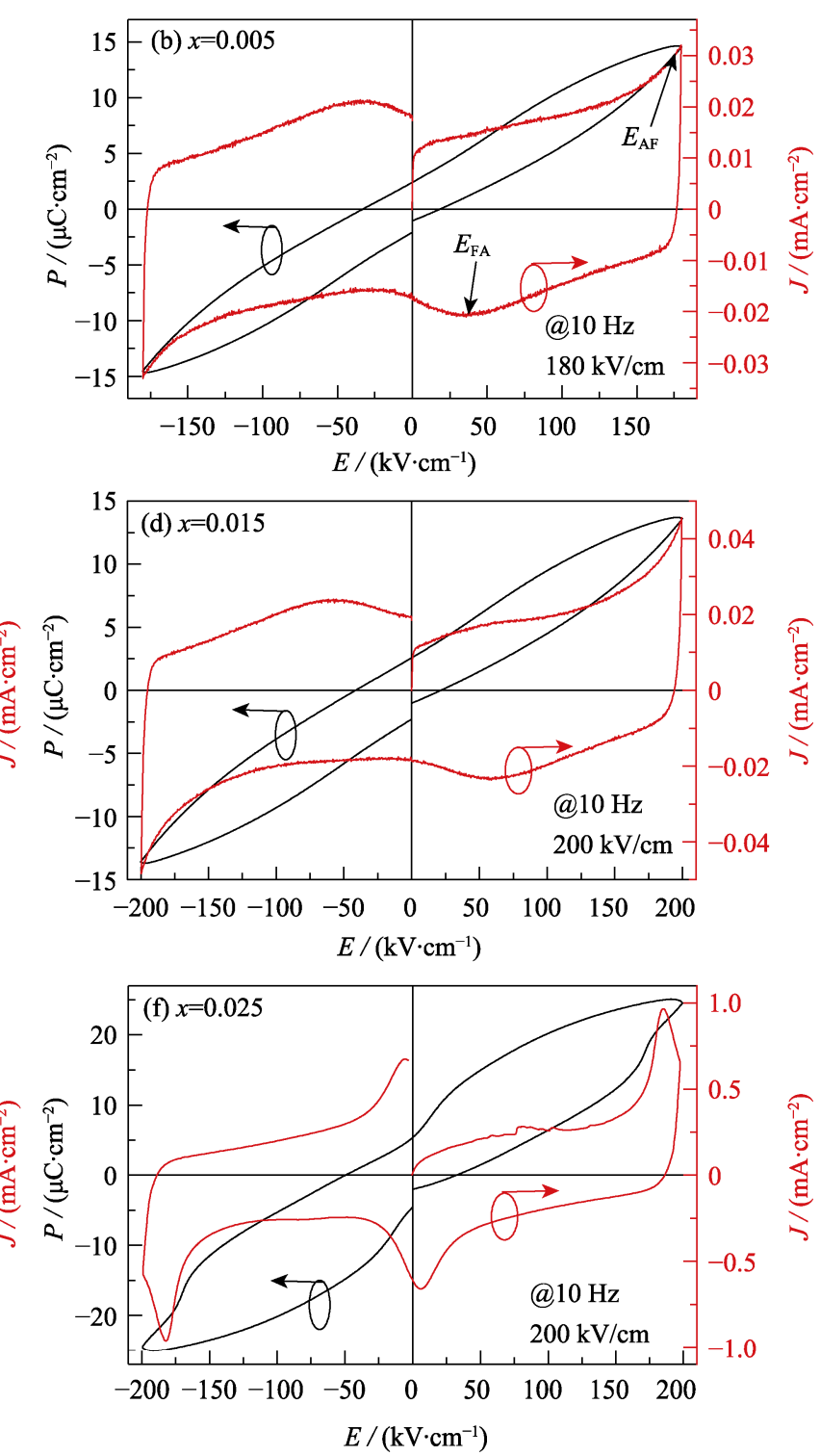

图 $6 \mathrm{NNCZ}-x \mathrm{Fe}$ 陶瓷室温下测得的电滞回线

Fig. $6 \quad P-E$ loops of NNCZ- $x \mathrm{Fe}$ ceramics at room temperature (a) $x=0$; (b) $x=0.005$; (c) $x=0.01$; (d) $x=0.015$; (e) $x=0.02$; (f) $x=0.025$

对 $x=0.02$ 样品的温度稳定性和频率稳定性作进一 步研究。

图 8 是 NNCZ-0.02Fe 陶瓷在不同温度和不同频 率的电滞回线和储能性能。由图 $8(\mathrm{a}, \mathrm{b})$ 可见, 随着 温度升高, NNCZ-0.02Fe 样品的电滞回线逐渐展开, 对应的 $W$ 和 $W_{\mathrm{rec}}$ 逐渐升高, 室温至 $100{ }^{\circ} \mathrm{C}$ 表现较好 的温度稳定性, $\eta$ 变化率为 $7.3 \%$ 。在 $125{ }^{\circ} \mathrm{C}$ 下 $W$ 和 $W_{\mathrm{rec}}$ 分别为 4.53 和 $1.53 \mathrm{~J} / \mathrm{cm}^{3}$, 但是 $\eta$ 下降到 $33.8 \%$ 。 从图 8(a)可知, 其双电滞回线突变成哑铃状, 表明 在 $125{ }^{\circ} \mathrm{C}$ 和 $180 \mathrm{kV} / \mathrm{cm}$ 电场强度下, 大量的 $\mathrm{AFE}$ 向 $\mathrm{FE}$ 发生了转变导致 $P_{\max }$ 和 $W$ 急剧增大。

NNCZ-0.02Fe 陶瓷的储能性能在 $10 \sim 100 \mathrm{~Hz}$ 范 围内表现出良好的稳定性, 随着频率增加, $\eta$ 由
$63.5 \%$ 增大到 $66.5 \%, \eta$ 变化率为 $4.7 \%$, 这是由于 $\mathrm{NNCZ}-0.02 \mathrm{Fe}$ 陶瓷内的畴翻转跟不上外部电场, 所 以 $W$ 稍微减小, $W_{\mathrm{rec}}$ 基本保持不变, $\eta$ 有上升趋势 ${ }^{[31]}$ 。

\section{3 结论}

通过传统固相法制备的 $\left(0.96 \mathrm{NaNbO}_{3}\right.$ $\left.0.04 \mathrm{CaZrO}_{3}\right)-x \mathrm{Fe}_{2} \mathrm{O}_{3}(\mathrm{NNCZ}-x \mathrm{Fe})$ 陶瓷，呈现单一钙 钛矿结构, 无明显的第二相。掺杂 $\mathrm{Fe}_{2} \mathrm{O}_{3}$ 能明显降 低 NNCZ 陶瓷的烧结温度, 且随着掺杂量增大, 晶 粒的平均尺寸和介电常数先减小后增大, $x=0.02$ 时, 样品的平均晶粒尺寸最小 $(5.04 \mu \mathrm{m})$, 击穿强度最大 $(230 \mathrm{kV} / \mathrm{cm})$, 介电常数最高。室温下, NNCZ-0.02Fe 

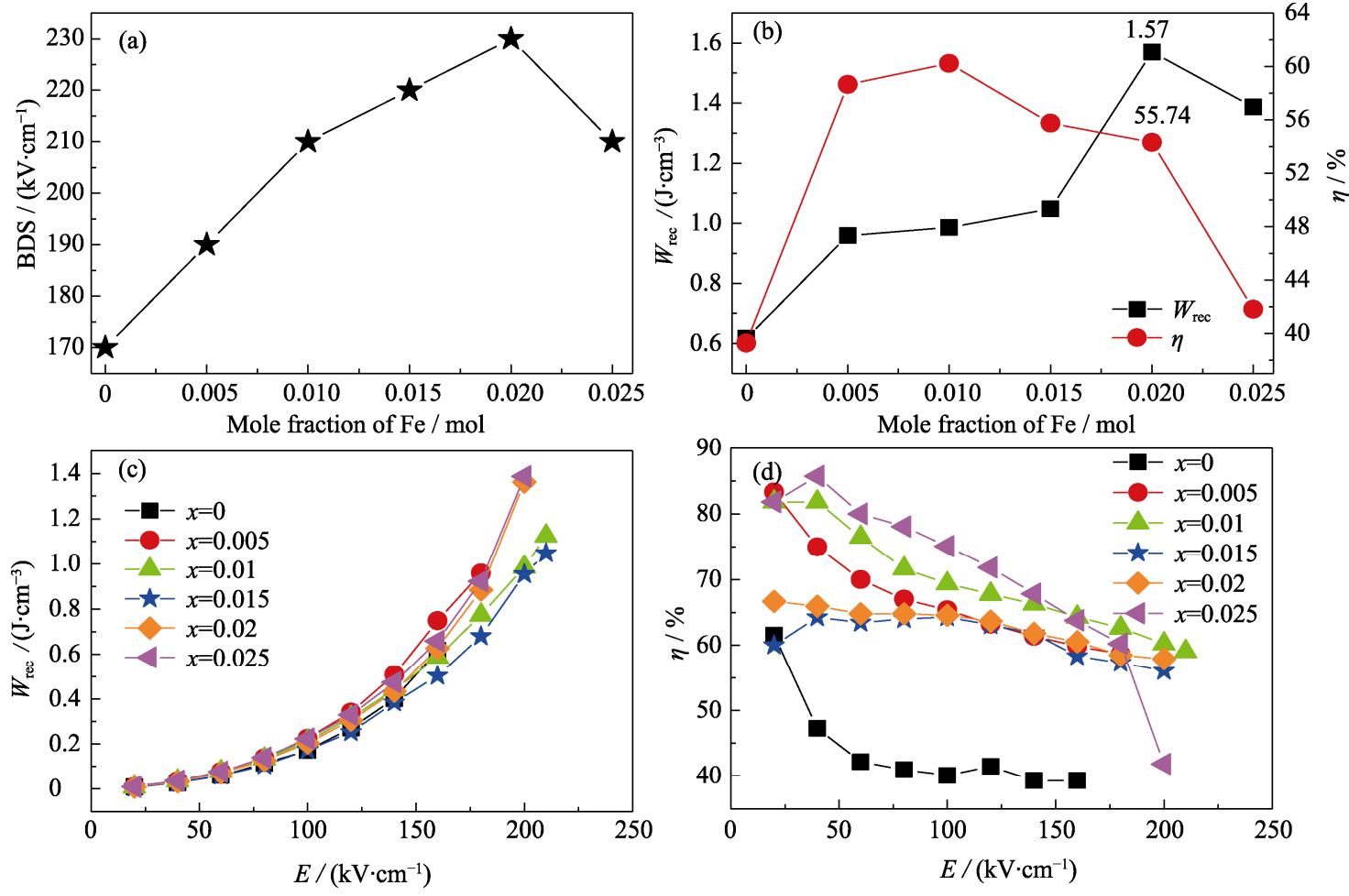

图 7 NNCZ- $x \mathrm{Fe}$ 陶瓷的击穿强度和储能性能

Fig. 7 Breakdown strength and energy storage property of NNCZ-xFe ceramics

(a) Breakdown strength; (b) Energy storage property before breakdown; (c, d) Energy storage property at different electric fields
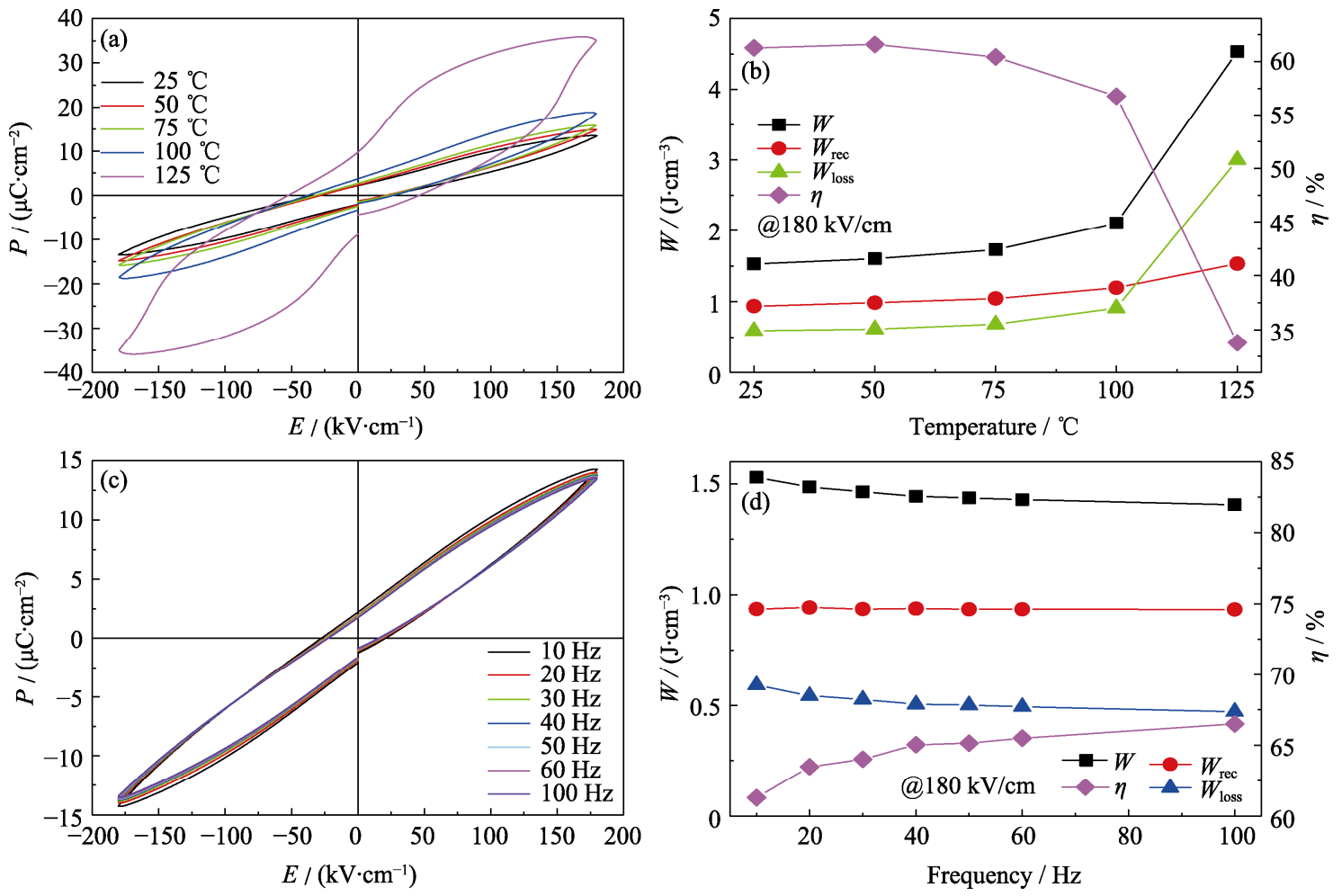

图 8 NNCZ-0.02Fe 陶瓷在不同温度和频率下的电滞回线储能性能

Fig. $8 \quad P$-E loops and energy storage properties of NNCZ-0.02Fe ceramics at different temperatures and different frequencies

(a) $P$-E loops of different temperatures; (b) Energy storage properties of different temperatures; (c) $P$ - $E$ loops of different frequencies;

(d) Energy storage properties of different frequencies

Colorful figures are available on website 
陶瓷击穿前的有效储能密度 $\left(W_{\mathrm{rec}}\right)$ 和储能效率 $(\eta)$ 分 别为 $1.57 \mathrm{~J} / \mathrm{cm}^{3}$ 和 $55.74 \%$ 。在电场强度 $180 \mathrm{kV} / \mathrm{cm}$ 下, 室温至 $100{ }^{\circ} \mathrm{C}, \mathrm{NNCZ}-0.02 \mathrm{Fe}$ 表现出良好的温 度稳定性, $\eta$ 变化率为 $7.3 \%$, 在 $125{ }^{\circ} \mathrm{C}$ 下, 储能密度 为 $4.53 \mathrm{~J} / \mathrm{cm}^{3}$; 在测试频率为 $10 \sim 100 \mathrm{~Hz}$ 时, 表现较 高且稳定的储能效率，储能效率维持 $63.5 \%$ 以上, $\eta$ 变化率为 $4.7 \%$ 。本研究表明: NNCZ-0.02Fe 在电介 质储能领域具有较好的潜在应用价值。

\section{参考文献:}

[1] QI H, ZUO R Z. Linear-like lead-free relaxor antiferroelectric $\left(\mathrm{Bi}_{0.5} \mathrm{Na}_{0.5}\right) \mathrm{TiO}_{3}-\mathrm{NaNbO}_{3}$ with giant energy-storage density/efficiency and super stability against temperature and frequency. J. Mater. Chem. A, 2019, 7(8): 3971-3978.

[2] LIU Z Y, LU J S, MAO Y Q, et al. Energy storage properties of $\mathrm{NaNbO}_{3}-\mathrm{CaZrO}_{3}$ ceramics with coexistence of ferroelectric and antiferroelectric phases. J. Eur. Ceram. Soc., 2018, 38(15): 4939-4945.

[3] ZHU L F, YAN Y K, LENG H Y, et al. Energy-storage performance of $\mathrm{NaNbO}_{3}$ based multilayered capacitors. J. Mater. Chem. C, 2021, 9(25): 7950-7957.

[4] SHIMIZU H, GUO H Z, REYES-LILlO S E, et al. Lead-free antiferroelectric: $x \mathrm{CaZrO}_{3}-(1-x) \mathrm{NaNbO}_{3}$ system $(0 \leqslant x \leqslant 0.10)$. Dalton. T., 2015, 44(23): 10763-10772.

[5] LIU X, ZHAO Y Y. Research progress of antiferroelectric energy storage ceramics. Electronic Components and Materials, 2020, 39(11): 55-66.

[6] MATTHIAS B T. New ferroelectric crystals. Physical Review, 1949, 75(11): 1771.

[7] VOUSDEN P. The non-polarity of sodium niobate. Acta. Cryst., 1952, 5(5): 690.

[8] ZHANG H F, YANG B, YAN H X, et al. Isolation of a ferroelectric intermediate phase in antiferroelectric dense sodium niobate ceramics. Acta Mater. 2019, 179: 255-261.

[9] GUO H Z, SHIMIZU H, RANDALL C A. Microstructural evolution in $\mathrm{NaNbO}_{3}$-based antiferroelectrics. J. Appl. Phys., 2015, 118(17): 174107.

[10] GUO H Z, SHIMIZU H, RANDALl C A. Direct evidence of an incommensurate phase in $\mathrm{NaNbO}_{3}$ and its implication in $\mathrm{NaNbO}_{3}$-based lead-free antiferroelectrics. Appl. Phys. Lett., 2015, 107(11): 112904.

[11] GAO L S, GUO H Z, ZHANG $\mathrm{S} \mathrm{J}$, et al. Stabilized antiferroelectricity in $x \mathrm{BiScO}_{3}-(1-x) \mathrm{NaNbO}_{3}$ lead-free ceramics with established double hysteresis loops. Appl. Phys. Lett., 2018, 112(9): 092905.

[12] GUO H Z, SHIMIZU H, MIZUNO Y, et al. Strategy for stabilization of the antiferroelectric phase (Pbma) over the metastable ferroelectric phase $\left(\mathrm{P}_{1}{ }_{1} \mathrm{ma}\right)$ to establish double loop hysteresis in lead-free (1-x) $\mathrm{NaNbO}_{3}-x \mathrm{SrZrO}_{3}$ solid solution. J. Appl. Phys., 2015, 117(21): 214103.

[13] GAO L S, GUO H Z, ZHANG S J, et al. A perovskite lead-free antiferroelectric $x \mathrm{CaHfO}_{3}-(1-x) \mathrm{NaNbO}_{3}$ with induced double hysteresis loops at room temperature. J. Appl. Phys., 2016, 120(20): 204102.

[14] QI H, ZUO R Z, XIE A W, et al. Excellent energy-storage properties of $\mathrm{NaNbO}_{3}$-based lead-free antiferroelectric orthorhombic P-phase (Pbma) ceramics with repeatable double polarization-field loops. J. Eur. Ceram. Soc., 2019, 39(13): 3703-3709.

[15] YE J M, WANG G S, CHEN X F, et al. Enhanced antiferroelectricity and double hysteresis loop observed in lead-free (1-x) $\mathrm{NaNbO}_{3}-x \mathrm{CaSnO}_{3}$ ceramics. J. Appl. Phys., 2019, 114(12): 122901.

[16] YE J M, WANG G S, CHEN X F, et al. Effect of rare-earth doping on the dielectric property and polarization behavior of antiferroelectric sodium niobate-based ceramics. J. Materiomics, 2021, 7(2): 339-346.

[17] ZHAO L, LIU Q, ZHANG S J, et al. Lead-free $\mathrm{AgNbO}_{3}$ antiferroelectric ceramics with an enhanced energy storage performance using $\mathrm{MnO}_{2}$ modification. J. Mater. Chem. C, 2016, 4(36): 8380-8384.

[18] WOLSKA A, MOLAK A, LAWNICZAK-JABLONSKA K, et al. XANES Mn K edge in $\mathrm{NaNbO}_{3}$ based ceramics doped with $\mathrm{Mn}$ and Bi ions. Phys. Scripta, 2005, 2005(T115): 989-991.

[19] CHAO L M, HOU Y D, ZHENG M P, et al. $\mathrm{NaNbO}_{3}$ nanoparticles: rapid mechanochemical synthesis and high densification behavior. J. Alloy. Compd., 2017, 695: 3331-3338.

[20] DONG L, DONG G X, ZHANG Q. Dielectric properties of $\mathrm{Fe}_{2} \mathrm{O}_{3}$-doped $\mathrm{MgTiO}_{3}-\mathrm{CaTiO}_{3}$ microwave ceramics. Materials Review, 2016, 30(5): 47-50.

[21] WANG X, REN P R, REN D, et al. B-site acceptor doped $\mathrm{AgNbO}_{3}$ lead-free antiferroelectric ceramics: the role of dopant on microstructure and breakdown strength. Ceram. Int., 2020, 47(3): 3699-3705.

[22] KANG H B, CHANG J Y, KOH K, et al. High quality Mn-doped $(\mathrm{Na}, \mathrm{K}) \mathrm{NbO}_{3}$ nanofibers for flexible piezoelectric nanogenerators. ACS Appl. Mater. Inter., 2014, 6(13): 10576-10582

[23] YANG B, BIAN J, WANG L, et al. Enhanced photocatalytic activity of perovskite $\mathrm{NaNbO}_{3}$ by oxygen vacancy engineering. Phys. Chem. Chem. Phys., 2019, 21(22): 11697-11704.

[24] ALLEN G C, BUTLER I S, KIRBY C. Characterization of ferrocene and ( $\eta^{6}$-benzene) tricarbonylchromium complexes by X-ray photoelectron spectroscopy. Inorg. Chim. Acta, 1987, 134: 289-292.

[25] YAN X D, ZHENG M P, ZHU M K, et al. Enhanced electrical resistivity and mechanical properties in BCTZ-based composite ceramic. J. Adv. Dielect., 2019, 9: 1950036.

[26] JIANG C B, MA C, LUO K H, et al. Piezoelectric and ferroelectric properties of $\mathrm{Na}_{0.5} \mathrm{Bi}_{4.5} \mathrm{Ti}_{4} \mathrm{O}_{15}-\mathrm{BaTiO}_{3}$ composite ceramics with $\mathrm{Mg}$ doping. J. Adv. Dielect., 2019, 9: 1950005.

[27] HU H, JIANG X P, CHEN C, et al. Influence of $\mathrm{Ce}^{3+}$ substitution on the structure and electrical characteristics of bismuth-layer $\mathrm{Na}_{0.5} \mathrm{Bi}_{8.5} \mathrm{Ti}_{7} \mathrm{O}_{27}$ ceramics. J. Inorg. Mater., 2019, 34(9): 997-1003.

[28] SHANNON R D, FISCHER R X. Empirical electronic polarizabilities in oxides, hydroxides, oxyfluorides, and oxychlorides. Phys. Rev. B, 2006, 73: 235111.

[29] YANG L T, KONG X, LI F, et al. Perovskite lead-free dielectrics for energy storage applications. Prog. Mater. Sci., 2019, 102: 72108.

[30] WANG T, WANG Y H, YANG H B, et al. Dielectric and energy storage property of $\mathrm{BaTiO}_{3}-\mathrm{ZnNb}_{2} \mathrm{O}_{6}$ ceramics. J. Inorg. Mater, 2019, 35(4): 431-438.

[31] DU J H, LI Y, SUN N N, et al. Dielectric, ferroelectric and high energy storage behavior of $(1-x) \mathrm{K}_{0.5} \mathrm{Na}_{0.5} \mathrm{NbO}_{3}-x \mathrm{Bi}\left(\mathrm{Mg}_{0.5} \mathrm{Ti}_{0.5}\right) \mathrm{O}_{3}$ lead free relaxor ferroelectric ceramics. Acta Phys. Sin., 2020, 69(12): 127703. 